\title{
La nutria marina (Lontra felina) en los Andes del sur del Perú
}

\author{
Sea otter (Lontra felina) in the Andes of southern Peru
}

1,a Joaquín A. Ugarte-Núñez

\section{NOTA CIENTÍFICA \\ ${ }^{1}$ Asociación para la conservación y el Desarrollo Sostenible. Sallqa, Perú \\ E-mail:jugarte@knighpiesold.com}

aRCID: 0000-0001-6298-1818

Palabras clave: Conservación, Cotahuasi y Colca, distribución fluvial, ríos andinos

Keywords: Conservation, Cotahuasi and Colca, riverine distribution, andean rivers

\section{Información adicional}

Presentado: 09/03/2021

Aprobado: 11/06/2021

\section{RESUMEN}

La nutria marina, gato marino o chungungo (Lontra felina) es considerada virtualmente una especie exclusiva marina con una preferencia de hábitat rocosos de litoral en la costa pacífica de Perú y hasta Tierra del Fuego al sur de Chile y la Isla de los Estados en el sur de Argentina. En esta nota científica se presentan registros fluviales de la nutria marina. En el río Cotahuasi (cuenca del río Ocoña) se tiene un registro hasta la localidad de Sipia, a $171 \mathrm{Km}$ del litoral y a $1994 \mathrm{~m}$ de altitud. En la cuenca del río Colca (cuenca Majes), el registro más alejado es en la laguna Mamacocha en la provincia de Caylloma, a $157 \mathrm{Km}$ del mar a una altitud de $1702 \mathrm{~m}$. Se presentan además otros registros directos e indirectos hasta la desembocadura de estas cuencas. El registro de esta especie en dos de los ríos más grandes del suroeste de Perú y no en los demás (departamentos de Moquegua y Tacna), posiblemente se deba a la configuración extremadamente encañonada, lo que puede representar un refugio para esta especie amenazada, además de tener una abundante oferta alimenticia. Se considera necesario incluir acciones de conservación en la distribución fluvial de la nutria de mar, debido a que actividades como la construcción de presas, afectarían significativamente su presencia en este espacio y hábitat de su distribución, en gran medida desconocido.

\section{ABSTRACT}

The sea otter, sea cat or chungungo (Lontra felina) is considered virtually an exclusive marine species with a preference for rocky littoral habitats along the Pacific coast of Peru and up to Tierra del Fuego in southern Chile and Isla de los Estados in southern Argentina. This scientific note presents fluvial records of the sea otter. In the Cotahuasi River (Ocoña River basin) there is a record up to the locality of Sipia, 171 $\mathrm{km}$ from the coast and at an altitude of 1,994 $\mathrm{m}$ above sea level. In the Colca river basin (Majes basin), the farthest record is in the Mamacocha lagoon in the province of Caylloma, $157 \mathrm{~km}$ from the sea and at an altitude of 1,702 meters. There are also other direct and indirect records up to the mouth of these basins. The record of this species in two of the largest rivers in southwestern Peru and not in the others (departments of Moquegua and Tacna) is possibly due to the extremely channeled configuration, which may represent a refuge for this endangered species, in addition to having an abundant food supply. It is considered necessary to include conservation actions in the fluvial distribution of the nutra de mar, because activities such as the construction of dams would significantly affect its presence in this space and habitat of its distribution, largely unknown. 
Lontra felina (nutria marina, gato marino o chungungo) es la especie más pequeña del género y la única nutria marina de la costa Pacífica de Sudamérica (Estes, 1989). Se distribuye en la región fría templada de la costa Pacífica de Perú a Chile, teniendo la mayor población en Chile (Yoxon y Yoxon, 2014). Valqui (2012) indica que el límite norte de su distribución es en Chimbote, en el departamento de Ancash (9¹0' S). Este límite norte es aceptado por la mayoría de autores, mientras que Schweigger (1964) reporta la especie más al norte en la isla Lobos de Tierra, en el departamento de Piura (6²7'S). Apaza y Romero (2012) realizan una revisión de los registros de su distribución para Perú, desde Punta Aguja $\left(05^{\circ} 47^{\prime} \mathrm{S}\right)$ en Piura, hasta la frontera con Chile $\left(18^{\circ} 21^{\prime} \mathrm{S}\right)$ en Tacna, confirmando el límite norte en Chimbote, poniendo en duda el reporte en la isla Lobos de Tierra (Schweigger, 1964), y considerando el registro de Alfaro-Shigueto et al. (2011) en el puerto artesanal de Huanchaco (08 $04^{\prime}$ S) y el de Santillán y Caro (2007) en Puerto Salaverry ( $\left.8^{\circ} 13^{\prime} \mathrm{S}\right)$, ambos en el departamento de La Libertad, como registros marginales en la zona de transición entre hábitat óptimo al sur y falta de este al norte. Estas últimas localidades constituirían el límite norte actual con registros de avistamiento, con una posible distribución marginal más al norte o eventos ocasionales de colonización (Valqui, 2012). Los registros hacia el sur muestran como límite la Tierra del Fuego al sur de Chile y la isla de los Estados (54\%48'S) en el sur de Argentina (Larivière, 1998).

L. felina virtualmente es considerada una especie exclusiva marina con una preferencia de hábitat rocoso del litoral (Eiseberg y Redford, 1999; Boher, 2005; Córdoba et al., 2009; Valqui, 2010; Vianna et al., 2010; Yoxon y Yoxon, 2014, Sanino y Meza 2016). De acuerdo con Redford y Eisenberg (1992), Vianna et al. (2010) y Valqui (2012), L. felina puede estar ausente en varios cientos de kilómetros de costa en su rango de distribución debido a que su hábitat está fragmentado de forma natural en una alternancia muy heterogénea de hábitat considerado por los autores como adecuado (parches rocosos con cuevas, a veces muelles, naufragios o botes de pesca abandonados) y hábitats inadecuados como playas arenosas o litorales rocosos sin cuevas. Por otra parte, Rozzi \& Torres-Mura (1990) describen a la especie utilizando playas de arena, pero que en la actualidad esta especie se asocia a fragmentos de litoral rocoso expuesto, explicando este cambio en el uso de hábitats por el aumento de la ocupación, utilización y explotación humana de los recursos que ofrece el litoral.

Otros autores mencionan la presencia de L. felina en estuarios y algunos metros río adentro (Estes, 1986). Hvindberg-Hansen (1970), Tello (1972), Viacava et al. (1978), y Brownell (1978), reportan avistamientos de nutria en los ríos Camaná y Ocoña, en los que se alimentaba de camarones, hasta $40 \mathrm{Km}$ de distancia de la costa y a $650 \mathrm{~m}$ de altitud (cabe anotar que esta altitud se presenta en el río Majes a los $75 \mathrm{Km}$ de distancia del mar). Apaza et al. (2004) consideran que estos registros ya no ocurren debido al crecimiento de la presencia humana y al incremento de la vigilancia en la cría del camarón, lo que presumiblemente causa la presencia actual de L. felina predominantemente en zonas marinas.

Desde el año de 1997 hasta la actualidad, el autor ha realizado 13 registros directos e indirectos de $L$. felina (Tabla 1) en las partes andinas medias y bajas de dos cuencas del suroeste de Perú que nacen por deshielos en los altos Andes ( $>5000 \mathrm{~m}$ ) y que representan los cañones más profundos de América (National Geographic, 2017). La primera es la cuenca del río Cotahuasi que luego forma el río Ocoña, y la segunda, la cuenca del río Colca que forma aguas abajo al río Majes. Estos ríos desembocan en el Pacífico en el departamento de Arequipa (Figura 1). 


\section{Tabla 1}

Registros fluviales de Lontra felina en el surostes de Perú.

\begin{tabular}{|r|l|l|l|c|c|c|c|}
\hline & $\begin{array}{c}\text { Localidad de } \\
\text { registro }\end{array}$ & Provincia & Tipo de registro & $\begin{array}{c}\text { Año de } \\
\text { registro }\end{array}$ & Coord. sur & Coord. oeste & $\begin{array}{c}\text { Altitud } \\
\text { (msnm) }\end{array}$ \\
\hline 1 & Sipia & La Unión & Avistamiento/Piel & 1999 & $15^{\circ} 14^{\prime} 32.29^{\prime \prime} \mathrm{S}$ & $72^{\circ} 58^{\prime} 2.95^{\prime \prime} \mathrm{O}$ & 1983 \\
\hline 2 & Chaupo & La Unión & Entrevista & 2018 & $15^{\circ} 15^{\prime} 4.44^{\prime \prime} \mathrm{S}$ & $72^{\circ} 59^{\prime} 31.62^{\prime \prime} \mathrm{O}$ & 1852 \\
\hline 3 & Velinga & La Unión & Avistamiento & 1997 & $15^{\circ} 16^{\prime} 43.41^{\prime \prime} \mathrm{S}$ & $73^{\circ} 1^{\prime} 45.01^{\prime \prime} \mathrm{O}$ & 1705 \\
\hline 4 & Chaucalla & La Unión & Entrev./Disecado & 1997 & $15^{\circ} 35^{\prime} 12.69^{\prime \prime} \mathrm{S}$ & $73^{\circ} 5^{\prime} 49.14^{\prime \prime} \mathrm{O}$ & 889 \\
\hline & $\begin{array}{l}\text { Lamapampa- } \\
\text { Chillihuay }\end{array}$ & Condesuyos & Avistamiento & 1999 & $15^{\circ} 40^{\prime} 30.03^{\prime \prime} \mathrm{S}$ & $73^{\circ} 3^{\prime} 38.24^{\prime \prime} \mathrm{O}$ & 773 \\
\hline 6 & La Barrera & Condesuyos & Entrevista & 1999 & $15^{\circ} 46^{\prime} 54.51^{\prime \prime} \mathrm{S}$ & $73^{\circ} 5^{\prime} 3.87^{\prime \prime} \mathrm{O}$ & 652 \\
\hline 7 & Chiguay & Camaná & Avistamiento & 2016 & $16^{\circ} 20^{\prime} 40.07^{\prime \prime} \mathrm{S}$ & $73^{\circ} 8^{\prime} 26.29^{\prime \prime} \mathrm{O}$ & 70 \\
\hline & Laguna & & & & & & \\
8 & Mamacocha & Caylloma & Avistamiento & 2015 & $15^{\circ} 40^{\prime} 39.21^{\prime \prime} \mathrm{S}$ & $72^{\circ} 14^{\prime} 42.44 " \mathrm{O}$ & 1694 \\
\hline 9 & Andamayo & Castilla & Entrevista & 2014 & $15^{\circ} 51^{\prime} 18.91^{\prime \prime} \mathrm{S}$ & $72^{\circ} 26^{\prime} 44.53^{\prime \prime} \mathrm{O}$ & 886 \\
\hline 10 & Ongoro & Castilla & Entrevista & 2014 & $15^{\circ} 59^{\prime} 17.91^{\prime \prime} \mathrm{S}$ & $72^{\circ} 27^{\prime} 59.08^{\prime \prime} \mathrm{O}$ & 713 \\
\hline 11 & Aplao & Castilla & Entrevista & 2014 & $16^{\circ} 4^{\prime} 39.72^{\prime \prime} \mathrm{S}$ & $72^{\circ} 28^{\prime} 54.77^{\prime \prime} \mathrm{O}$ & 609 \\
\hline 12 & Characta & Camaná & Entrevista & 2014 & $16^{\circ} 30^{\prime} 35.533^{\prime \prime} \mathrm{S}$ & $72^{\circ} 38^{\prime} 28.09^{\prime \prime} \mathrm{O}$ & 114 \\
\hline 13 & Quilca & Camaná & Pers. Com. & 2017 & $16^{\circ} 37^{\prime} 22.29^{\prime \prime} \mathrm{S}$ & $72^{\circ} 18^{\prime} 14.35^{\prime \prime} \mathrm{O}$ & 212 \\
\hline
\end{tabular}




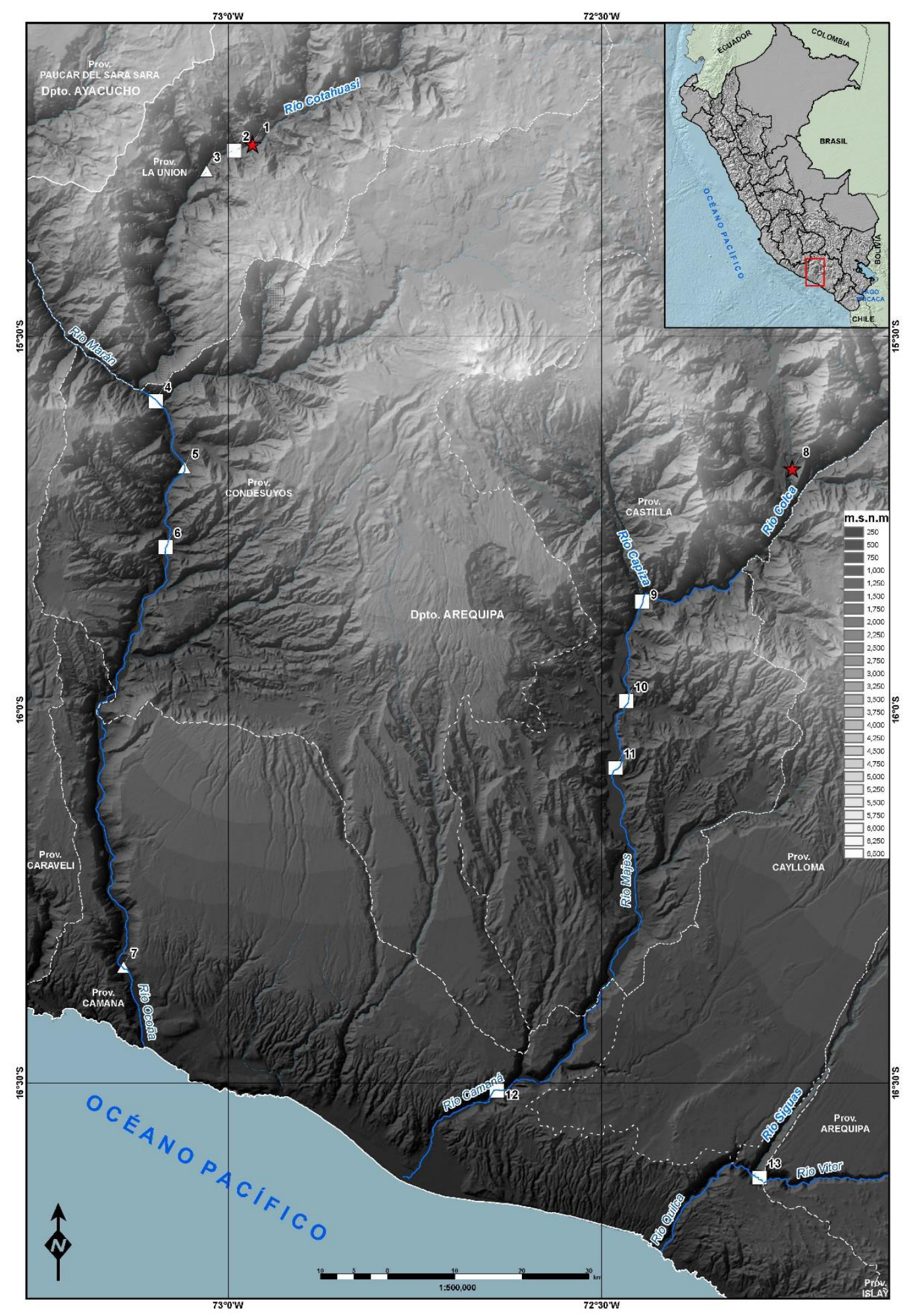

Figura 1. Ubicación de los registros fluviales de Lontra felina en el Suroeste de Perú. Las estrellas rojas representan los registros por avistamiento más alejados del litoral; los triándulos blancos, otro registros por avistameinto; $\mathrm{y}$, los cuadrados blancos, los registros indirectos (etrevistas, pieles).

En el río Cotahuasi, que se encuentra íntegramente en la provincia andina La Unión, se han realizado 4 registros, siendo el de la localidad de Sipia, a $171 \mathrm{Km}$ del litoral del Pacífico y a $1994 \mathrm{~m}$ de altitud al pie de 
la catarata de Sipia (la que posiblemente sea una barrera natural), el registro más alto y alejado del litoral. En la cuenca del Majes-Colca, de los 5 registros obtenidos, el de la laguna Mamacocha en la provincia de Caylloma es el que se aleja hasta $157 \mathrm{Km}$ del mar y llega a una altura de $1702 \mathrm{~m}$, cuyas aguas fluyen hacia el río Colca. Posiblemente, la distribución de L. felina se extienda aguas arriba de este río, que en esta sección es inaccesible (Figura 2).

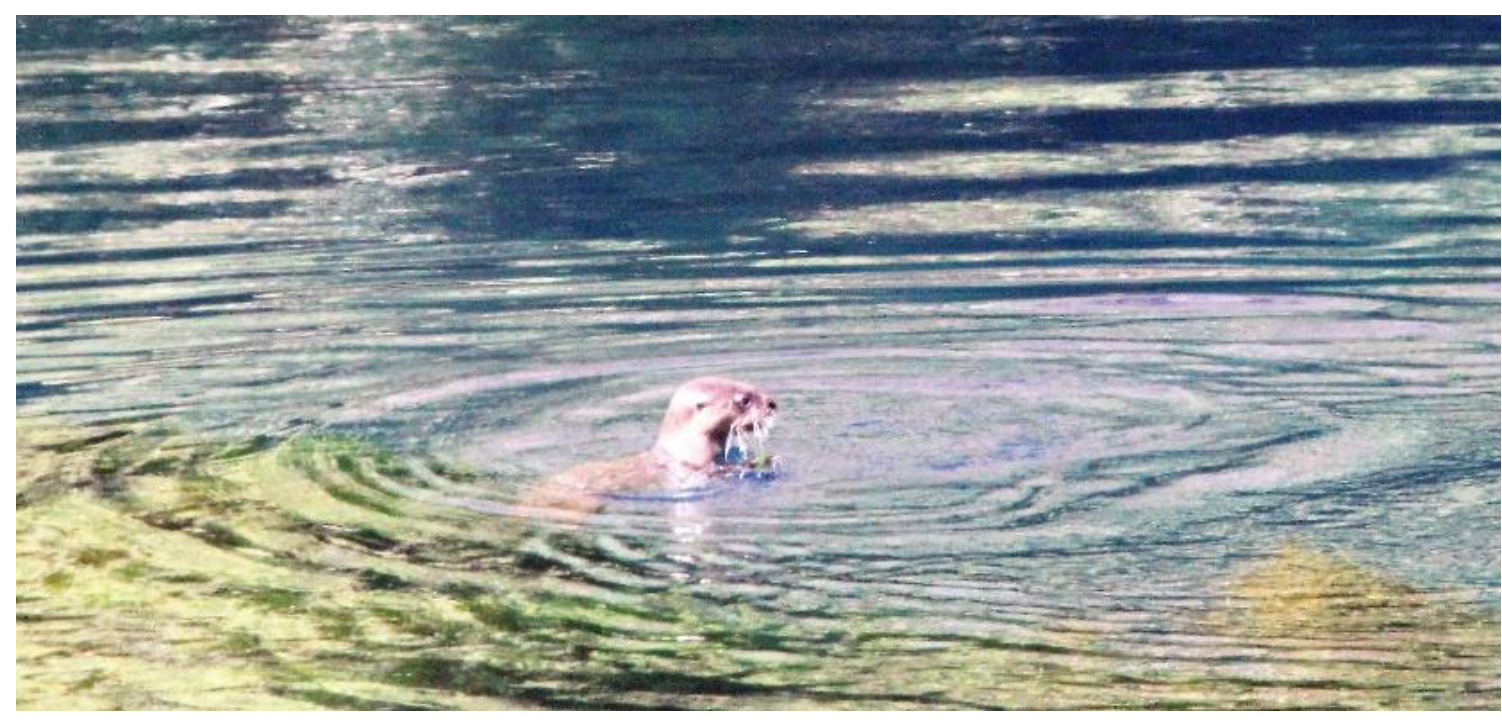

Figura 2. Individuo de Lontra felina en laguna Mamacocha (enero 2015)

Las localidades más alejadas del litoral tienen registros por avistamientos (Sipia, laguna Mamacocha) en las partes intermedias de las cuencas de los ríos Cotahuasi y Colca, así como en otras localidades río abajo (Velinga en la provincia La Unión, Lamapampa-Chillihuay en Condesuyos y Chiguay en la provincia de Camaná, todos en la cuenca del Cotahuasi-Ocoña).

En otras localidades se ha realizado el registro por entrevistas Ad libitum a los poblados, incluyendo Chaupo y Chaucalla en la cuenca del Cotahuasi, La Barrera en el río Ocoña y, Andamayo, Ongoro, Aplao y Characta en el río Majes (Figura 1). Se considera que esta especie no puede ser confundida con otra, debido a que es el único mamífero acuático en estas zonas, y por ser identificada históricamente como "Huallaque" en su distribución fluvial (V. Pacheco, pers com 1998). Los entrevistados indican que en las últimas décadas la cantidad de individuos observados se ha visto reducida, ya que en los años 50 remontaban el río por decenas. Incluso considerando la percepción de los entrevistados, ya que podrían sobreestimar el número de individuos reportados, se concluye que antes se avistaba a la especie con mayor frecuencia y en mayores cantidades. Los entrevistados en la localidad de Velinga de la cuenca del río Cotahuasi indican que la especie se reproduce en esa zona (1600 m s. n. m.), afirmando haber visto crías. Los pobladores de las partes cercanas al mar (Chiguay y Characta) indican que lo conocen como "Chungungo" y que más arriba, en los ríos, lo llaman "Huallaque".

No existen ríos en el suroeste de Perú hacia el norte o el sur de estas dos cuencas descritas previamente, que muestren características similares de caudal o que sean permanentes donde se haya registrado la especie. Sin embargo, hacia el sur, existe un registro (pers. com. K. Mares, 2018) en el río Quilca (provincia de Camaná) en la confluencia de los ríos Vítor y Siguas. En el río Tambo (entre Moquegua y Arequipa) que tiene un caudal considerable y permanente, no existen registros.

Los entrevistados consideran a L. felina como una especie que compite con los pobladores por el recurso camarón (Cryphiops caementarius), que es una especie de crustáceo muy apreciada y consumida en los ríos costeros del sur de Perú. Debe considerarse que los ríos que tienen mayor población de camarón son el Majes- Colca y Ocoña- Cotahuasi, junto con el Tambo más al sur, debido a que los ríos llevan mayor caudal de agua (Pinazo et al., 2020). Esta percepción de la población ha provocado un comportamiento 
negativo hacia la nutria por parte de los pobladores de zonas aledañas al río y consumidores del camarón, lo que, sumado a la caza por la piel para tapetes (Chaucalla y Sipia) y otros objetos ornamentales, constituye un riesgo para la supervivencia de la especie en estas áreas.

Lontra felina está considerada en peligro de extinción (EN), según la Unión Internacional para Conservación de la Naturaleza (IUCN) y la legislación peruana en el decreto de categorización de especies amenazadas silvestres de Perú (D. S. 004-2014). De acuerdo con su distribución conocida, se encuentra en áreas naturales protegidas marinas (Reserva Nacional Sistema de Islas, Islotes y Puntas Guaneras, Reserva Nacional Paracas y Reserva Nacional de San Fernando) (Valqui \& Rheingantz, 2015), más no en áreas protegidas continentales como la Reserva Paisajística de la Subcuenca del Cotahuasi hasta estos registros, por lo que las medidas de conservación están aplicadas a enfrentar las amenazas en su distribución marina, más no fluvial.

Es muy probable que la distribución fluvial de L. felina en las cuencas de los ríos Cotahuasi y Colca, se deba a su configuración extremadamente encañonada y a la poca población humana presente, lo que brinda cierta protección a la especie, por lo que estas cuencas podrían constituir el último refugio de su distribución fluvial. Debe considerarse también la importancia de la abundancia de recursos alimenticios como lo son el camarón de río y posiblemente peces como truchas y pejerreyes. Estos factores, refugio y oferta de alimento, concuerdan con lo indicado por Bastidas et al. (2007) que indica que la nutria marina es una especie oportunista, que selecciona sus presas en función de su disponibilidad y no de su aporte energético; asimismo, la proximidad a sus presas y el grado de exposición del sitio de alimentación podrían ser factores significativos que determinen su distribución (Medina-Vogel et al., 2007; Villegas et al., 2007)

Cualquier intervención que interrumpa esta distribución fluvial y muy probable ruta de migración, o el establecimiento de esta especie gravemente amenazada y carismática, como por ejemplo la construcción de represas, podría tener un impacto muy significativo sobre estas poblaciones desconocidas, en términos de su ecología y biología en agua dulce.

Se recomienda realizar evaluaciones de sus poblaciones fluviales, movimientos de migración vertical, estudios de alimentación y reproducción, así como tomar medidas para reducir las amenazas identificadas, incluyendo la capacitación a los pobladores locales, y el manejo de obras de infraestructura en estas áreas.

\section{REFERENCIAS}

Alfaro-Shigueto, J., Valqui, J., \& Mantel, J. C. (2011). Nuevo Registro de la Nutria Marina Lontra felina (Molina, 1782) al Norte de su Distribución Actual. Ecol. Apl., 10(2), 87-91.

Apaza, M., Valqui, J., Mangel, J., Roca, M., Alfaro, J., Santillan, L., Perret, J. P., Onton, G., Castaneda, C., Munemura, G., \& Tovar, A. (2004). Estado de Conservación de Lontra felina (Molina, 1782) en la Costa Peruana. In: Reporte para la Comisión Permanente del Pacífico Sur, Lima.

Apaza, M., \& Romero, L. (2012). Distribución y observaciones sobre la población de la nutria marina Lontra felina (Molina 1782) en el Perú. Revista Peruana de Biologia, 19(3), 285-298. https://doi.org/10.15381/rpb.v19i3.1064

Bastidas, R., Rodríguez, D., Secchi, E., \& da Silva, V. (2007). Mamíferos Acuáticos de Sudamérica y Antártida. Vázquez, M. (ed.). Buenos Aires, República Argentina.

Boher, E. (2005). Ámbito de hogar y territorialidad del chungungo Lontra felina (Molina 1782) en el litoral central de Chile, Quintay (Memoria de Título para optar al Título de Médico Veterinario). Universidad Austral de Chile. Facultad de Ciencias Veterinarias. Valdivia

Brownell Jr., R. L. (1978). Ecology and conservation of the Marine Otter (Lutra felina). In: Duplaix, N. (Ed.), Otters-Proceedings of the First Working Meeting of the IUCN/SSC Otter Specialist Group. Paramarimbo, Suriname, pp. 104-106.

Córdova, O., J. R. Rau, Suazo, C. G., \& Arriagada, A. (2009). Estudio comparativo de la ecología alimentaria del depredador de alto nivel trófico Lontra felina (Molina, 1782) (Carnivora: Mustelidae) en Chile. Revista de Biología Marina y Oceanografía, 44(2), 429-438. 
Eisenberg, J. F., \& Redford, K. H. (1999). Mammals of the Neotropics: The Central Neotropics. Chicago, Illinois, USA: University of Chicago.

Estes, J.A. (1986). Marine otters and their environments. Ambio, 15, 181-183.

Hvindberg-Hansen, H. (1970). A survey of the coast otter (Lutra felina) Molina in the Peruvian rivers of Camana and Oco na. FAO-UNDP/SP, p. 116.

Larivière, S. (1998). Lontra felina. Mamm. Spec. 575, 1-9.

Medina-Vogel, G., Boher, F., Flores, G., Santibañez, A., \& Soto-Azat, C. (2007). Spacing behavior of marine otters (Lontra felina) in relation to land refuges and fishery waste in Central Chile. $J$. Mammalogy, 88, 487-494.

National Geographic. (2017). Los 8 cañones más grandes del planeta. https://viajes.nationalgeographic.com.es/a/8-canones-mas-grandes-planeta_9019/1

Pinazo, K., Incio, A., \& Campos, S. (2020). Pesquería y condición biológica del camarón Cryphiops caementarius (Molina, 1782) en ríos de Arequipa-Perú, 2016-2018. Bol Inst Mar Perú. 35(1), 37-48.

Redford, K. H., \& Eisenberg, J. F. (1992). Mammals of the Neotropics, The Southern Cone: Chile, Argentina, Uruguay, Paraguay. Chicago: University of Chicago Press.

Rozzi, R., \& Torres-Mura, J. C. (1990). Observaciones del Chungungo (Lutra felina) al sur de la Isla Grande de Chiloé: antecedentes para su conservación. Medio Ambiente, 11 (1), 24-28.

Santillán, L., \& Caro, K. (2007). Mamíferos Marinos en el Puerto de Salaverry y Zonas Adyacentes. Libro de resúmenes CONCIMAR.

Sanino, G., \& M. Meza. (2016). Ecología Trófica y Simpatría de Nutrias (Lontra felina y Lontra provocax) en la Reserva Añihué, Patagonia Chilena. Boletín del Museo Nacional de Historia Natural, (Boletín 65), 279-289. Disponible en http://publicaciones.mnhn.gob.cl/668/w3-article-73630.html

Schweigger, E. (1964). El litoral peruano. Lima: Universidad Nacional Federico Villareal.

Tello, E. (1972). Anotaciones Sobre el Camarón. Documenta. Min. Pesq. Lima. 18, 59.

Valqui, J., Hartl, G. B., \& Zachos, F. E. (2010). Non-invasive Genetic Analysis Reveals High Levels of mtDNA Variability in the Endangered South-American Marine Otter (Lontra Felina). Conservation Genetics, 11 (5), 2067-2072.https://doi.org/10.1007/s10592-010-0069-9.

Valqui, J. (2012). The Marine Otter Lontra felina (Molina, 1782): A Review of Its Present Status and Implications for Future Conservation. Mammalian Biology, 77; 75-83.

Valqui, J., \& Rheingantz, M. L. (2015). Lontra felina (errata version published in 2017). The IUCN Red List of Threatened Species 2015: e.T12303A117058682. https://doi.org/10.2305/IUCN.UK.20152.RLTS.T12303A21937779.en.

Viacava, C., Aitken, R., Llanos, J. (1978). Estudio del Camarón en el Perú: 1975-1976. Inst. Mar. del Perú. Bol. 3(5), 161-232.

Vianna, J., Ayerdi, P., Medina-Vogel, G., Mangel, J.C., Zeballos, H., Apaza, M., \& Faugeron, S. (2010). Phylogeography of the Marine Otter (Lontra felina): historical and contemporary factors determining its distribution. J. Hered., 101 (6), 676-689.

Villegas, M. J., Aron, A., \& Ebensperger, L. A. (2007). The influence of wave exposure on the foraging activity of marine otter, Lontra felina (Molina, 1782) (Carnivora: Mustelidae) in northern Chile. J. Ethol., 25, 281-286.

Yoxon, P., \& Yoxon, G. (2014). Otters of the World. Whittles Publishing. 